\title{
Towards an Harmonious and Integrated Management Approach for Lifecycle Planning
}

\author{
Frédéric Demoly^, Samuel Deniaud, and Samuel Gomes \\ IRTES-M3M, \\ Université de Technologie de Belfort-Montbéliard (UTBM), \\ 90010 Belfort Cedex, France \\ \{frederic.demoly, samuel.deniaud, samuel.gomes\}@utbm.fr
}

\begin{abstract}
The paper presents an initiative towards the harmonious and integrated management of lifecycle planning, such as assembly planning, disassembly planning, maintenance planning and so on. This stake currently meets industrial requirements and research issues, mainly in product lifecycle management, integrated design, and lifecycle engineering fields. The fact of managing $\mathrm{X}$ planning during product design requires therefore a complete understanding of the product lifecycle processes and various abstraction layers of data-information-knowledge. In such a way, the critical outcome is to ensure the development of well-balanced product by considering the rationale of lifecycle planning and managing its related data, information and knowledge.
\end{abstract}

Keywords: Product Lifecycle Management, Integrated Design, Lifecycle Planning, Closed-Loop PLM, Concurrent Engineering.

\section{Introduction: Current Stakes}

The current ultra-competitive context, in the manufacturing and energy industries, has raised challenging issues for improving the elicitation and use of intellectual heritage (i.e. knowledge, know-how, intents, competencies ... ) and business actors involved in the company. Here several phases of the product lifecycle need to be identified as strategic and critical to ensure the development and the delivery of well-balanced products. In other words, a well-balanced product can be considered as a product taking into account, consistently, all the constraints of its life cycle with an optimum cost (i.e. assembly-friendly products, green products, service-oriented products ...). At this stage, the proposed research project focuses on the following product lifecycle processes: product design and $\mathrm{X}$ planning definition where $\mathrm{X}$ stands for manufacturing, assembly, disassembly, maintenance, transport ...

At a critical place, the design phase requires the consideration and integration of all constraints and knowledge (business processes, business terms, expert rules, job experience ...) of product lifecycle phases. However, the consideration of a large amount of rules increases the work complexity of product architects and designers. An emerging way allows to overcome this issue differently: over the

\footnotetext{
* Corresponding author.
} 
past two decades, engineering paradigm has shift from sequential engineering to concurrent engineering, therefore facilitating the integration of specific concerns into product design stages [1. It seems that this shift has generated potential gains by using heuristics rules in addition to qualitative information (such as described in Fig. 1) 2. Today, the concurrent engineering philosophy has reached its limit. Indeed, current approaches, in the field of concurrent engineering, do not provide enough information layer for a full understanding of product architects and designers. In such a way, it is important to address a proactive vision of the product development by considering downstream processes as early as possible in product design (Fig. 1) [3]. Thus awareness and understanding will be promoted to product/process architects and designers. This can be done with relevant data input and some specific layers which may introduce qualitative context based on formal description (Fig. 1). Here qualitative context may include formal description of engineering intents and knowledge such as explained in 2 .

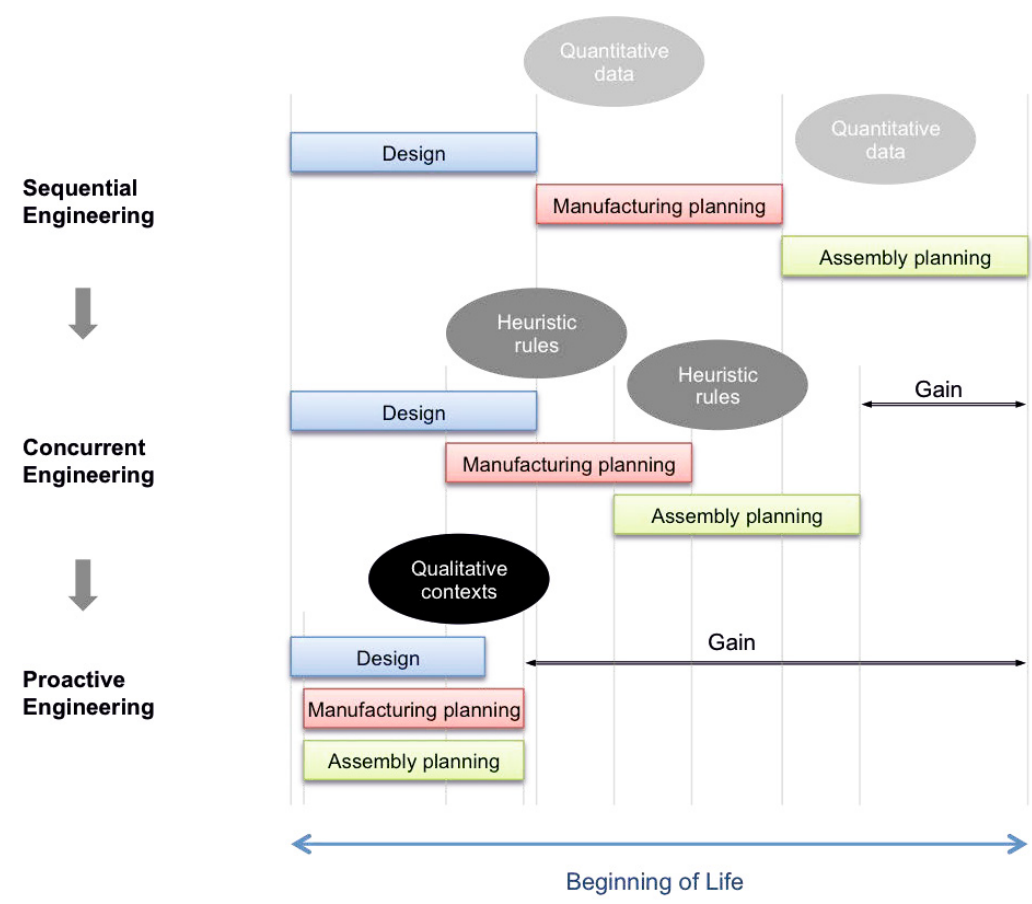

Fig. 1. Current stakes: From sequential to proactive engineering

\section{Description of the Research Project}

\subsection{Computational Intelligence}

The definition of lifecycle planning (i.e. manufacturing planning, assembly planning, maintenance planning, disassembly planning ...) by taking into account 
the appropriate knowledge, at the earlier stage of product design, enables the introduction of a context layer for architects and designers, as described with "The design context knowledge can be defined as the related surrounding knowledge of a design problem at a moment in time for given consideration" 6]. Recent research results have shown that it is possible to generate and define an assembly sequence before defining any product geometry of mechanical products, such as in the domains of automotive and aerospace industries 34. This early-defined assembly sequence has influenced the product design by structuring the product with various assembly levels and introducing skeleton entities related to product relationships [5].

The today's challenge of R\&D focuses not only on the definition of a single assembly plan but also requires now the incorporation of a wider range of lifecycle phases such as manufacturing planning, maintenance planning, transport planning, selective disassembly planning to name a few, and this needs to be considered in a harmonious and coherent manner (Fig. 2). To do this, a major effort should be made to elicit, centralize, nest constraints and expert rules, but also to relate it based on compromise at a strategic level.

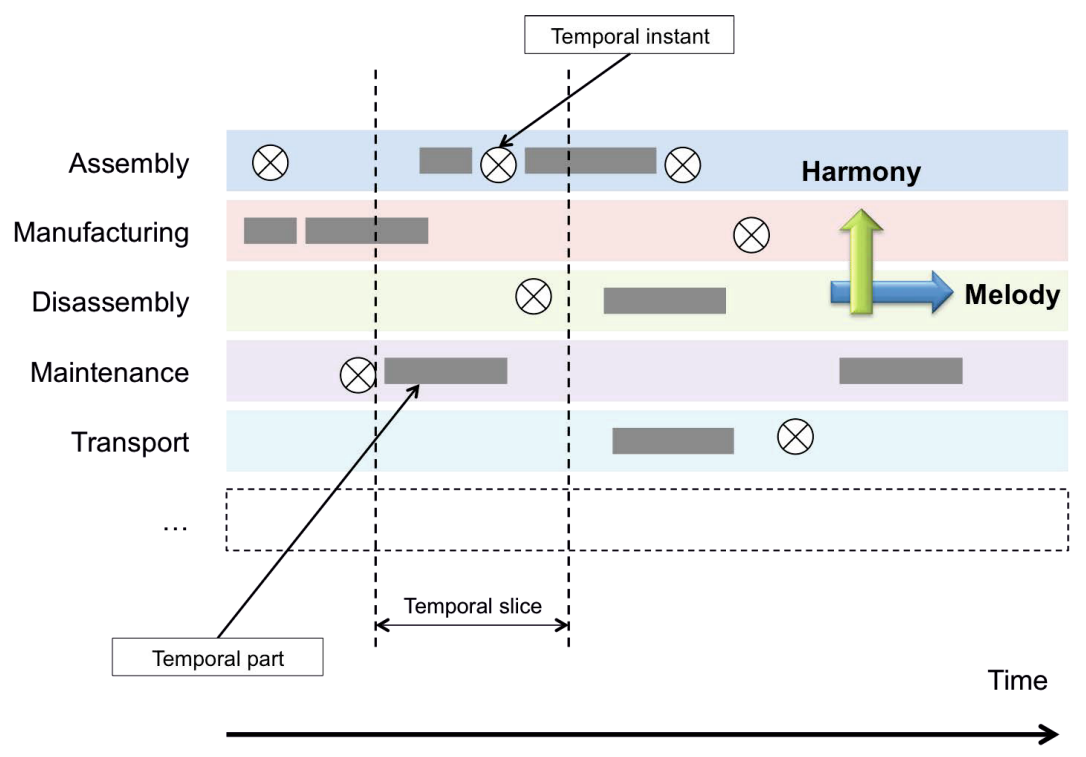

Fig. 2. Multiple life phase planning to be managed

On a more technical level, and regarding the data model, the research project will highlight a product-process ontology, in order to connect formally and semantically objects in space (parts, sub-assemblies, resources ...) and objects time (assembly, maintenance, disassembly and transport operations ... ). Based on this formal ontology, different approaches may be proposed. 
The first effort consists in generating all the admissible lifecycle plans from a minimal product model, the product-process ontology, expert rules and strategic constraints as quick as possible. This will require the development of mathematical algorithms based on patterns recognition matrix previously developed in collaboration with automotive industry [3. The generation of feasible solutions can be achieved automatically or semi-automatically, so that lifecycle planners will be able to control the definition of their sequences.

In order to control the combinational complexity, due to the number of operations generated in all lifecycle plans, a multi-level approach will be implemented to generate and break down these plans. Therefore the aim is to get high levels plans or sequences, also called - macro plans/sequences - almost invariant thus facilitating reuse and low levels plans/sequences, called - micro plans/sequences. Once all admissible plans have been generated, it will be necessary to identify the plans which meet the best engineering compromise. An additional effort will be added to allow the reconfiguration of lifecycle plans if an unpredictable event that may occurs to different phases of the life cycle (e.g. the reconfiguration of a selective disassembly sequence for maintenance in use). Consequently, the selection of the sequence/plan during product design and definition will have to take into account specific criteria aiming at selecting a sequence/plan while minimizing, on one hand the reconfiguration cases, and on the other hand giving multiple reconfigurations solutions if appropriate. The search of reconfigurations cases will be applied in a special way at micro sequences/plans level.

\subsection{Systems Engineering Applied to Multiple Sequence Planning}

In parallel to the generation of these lifecycle plans, a specific allocation of customer and lifecycle-oriented technical requirements will be addressed at various abstraction levels of the life phase plan. In order to address this requirement allocation activity, a framework is proposed as illustrated in Fig. 3. First an ontological level is done in order to define the different concepts and their relations. Second, these concepts and relations are expressed into specific diagrams that give different viewpoints about the lifecycle plans to the planners. Third, specific diagrams are built following a specific process to formalize the lifecycle plans of the system-of-interest. This process needs to investigate the necessary objects to realize the solution. They are characterized by various attributes captured at the data level. Language such System Modeling Language (SysML) 7] is a good candidate to express the needed concepts and relations in specific diagrams. It provides profile mechanism in order to specialize and extend the elements of the language.

A specific allocation of customer and lifecycle-oriented technical requirements will be addressed at various abstraction levels of the life phase plan (Fig. 4). At each decomposition level, activity diagram (act), internal bloc diagram (ibd) and requirement diagram (req) are modeled based on each operation and its attached requirements. The first one expresses the behaviour of the operation 


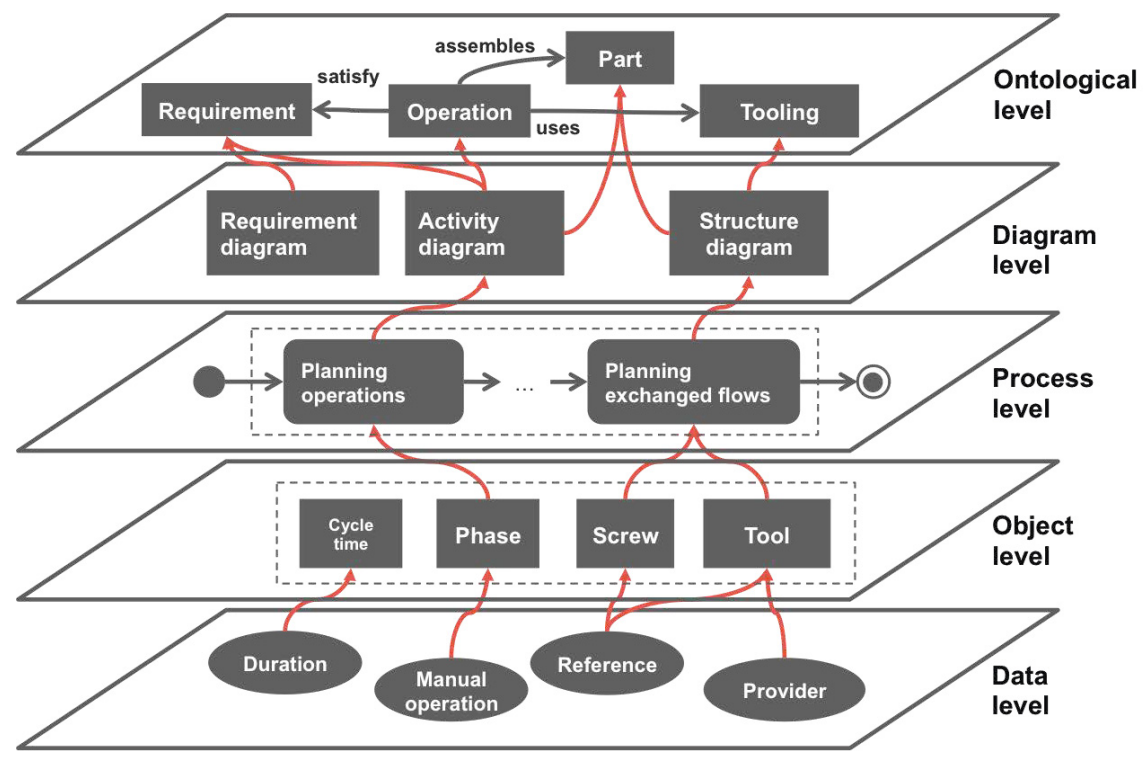

Fig. 3. Proposed framework

$i_{n+1}$ by decomposing it into several operations. The second one depicts the various flows of matters, energy and information exchanged by the operations and the environment of the operation $i_{n+1}$. Finally, the third one explains the decomposition of the requirements of the operation $i_{n+1}$ and their allocation links. Recursively, these three diagrams are modeled at the level $i_{n-1}$ respectively for the operations $1 n, 2 n$ and $3 n$.

In such a way the system engineering principles can be applied the generation of the lifecycle plans in order to master the complexity of such sequence planning. For instance, these allocation links will allow to perform impact analysis when requirements change. Needless to say, these links will also ensure traceability of decisions.

\subsection{Systems/Tools Implementation}

The set of all lifecycle sequences/plans (including operations) will highlight the need to be managed by data management system. This novel system will enable the access to all lifecycle planners in order to define constraints, operations, temporal logic ... A solution consists in proposing an approach for managing compromises between lifecycle plans and decisions made during the definition lifecycle plans. In addition and in order to ensure the digital chain covering the product lifecycle, the proposed platform will have to be integrated and connected to existing systems and tools of the company, such as PDM (Product Data Management), ERP (Enterprise Resource Planning), and CAD tools (Computer Aided Design). 


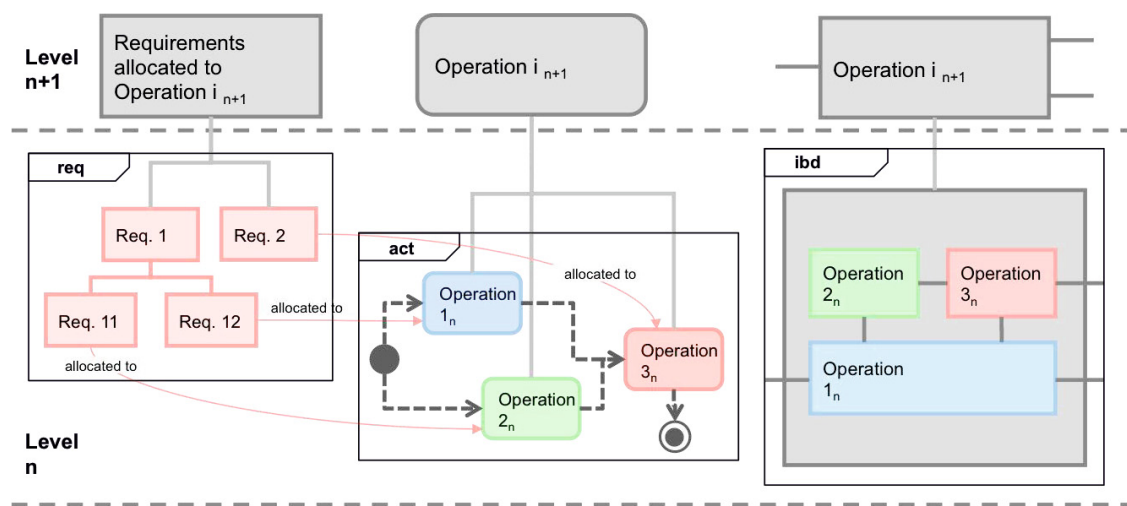

Level
n-1

Fig. 4. Diagrams defined at abstraction level

Currently, some commercial tools and systems close to the envisioned platform have been identified of which MPM (Manufacturing Process Management), but are only limited to the definition of manufacturing and assembly planning (welding, final assembly ... ) for the manufacturing phase of the product. It therefore becomes critical to expand the capability of these systems to the definition of all lifecycle planning such as required all along the product lifecycle, and the integration of expert knowledge and logical reasoning.

\subsection{Project Objectives}

As a consequence, the proposed research project mainly aims at:

- Developing a knowledge model called ontology, integrating formal descriptions of the product-process, applied to the domains of automotive and energy industries;

- Developing methods to define lifecycle plans (manufacturing, assembly, maintenance, disassembly, transportation ... );

- Proposing methods for managing lifecycle plans in a real-time manner by integrating the management of consents and compromises, in order to promote a smart, harmonious, dynamic and symphonic orchestration;

- Representing the lifecycle plans in the form of causal knowledge in order to create decision making supports for the design and geometric modeling phases;

- Specifying and developing a Web-based platform by considering initial efforts towards the development of PEGASUS tool which works at the interface of PDM, MPM and CAD systems 4]. 


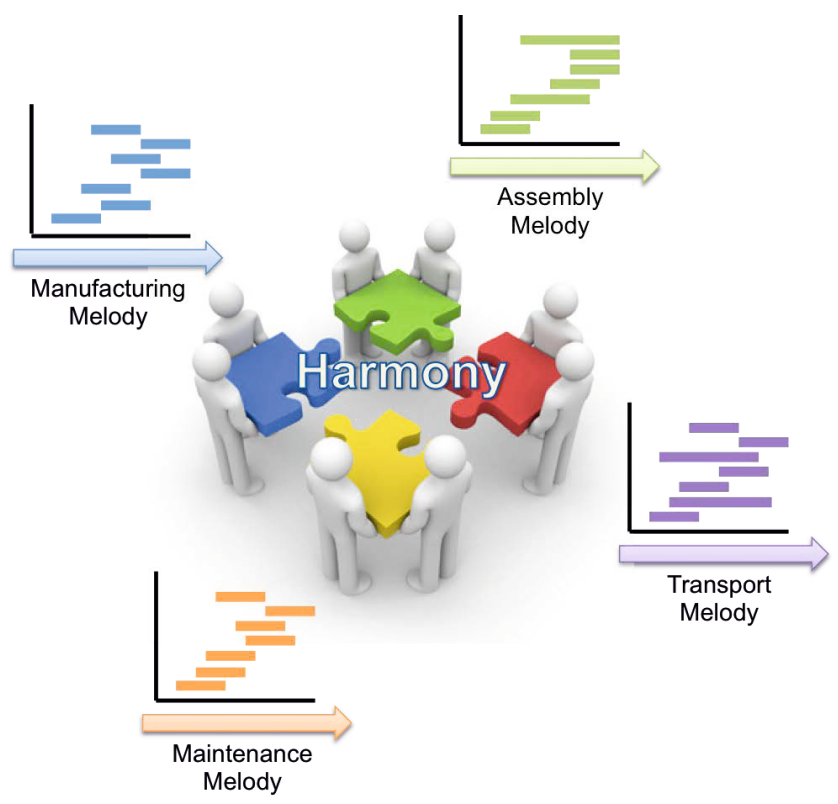

Fig. 5. Harmonious management of lifecycle process planning

\section{Synthesis}

This research initiative has been proposed by following an original analogy: the harmonious and integrated management of lifecycle plans can be considered as the management of symphonic orchestra. A symphony is an intelligent instrumental composition, with generally large proportions, and including several joints or disjointed movements, and using the resources of the symphony orchestra. Indeed, it is easy to compare between a sequence/plan and a musical partition. The originality here is to manage the sequence/plan of the melody (which means operations concatenation by respecting time as shown in Fig. 2) as well as harmony between the sequences/plans (which means operations superposition during a period of time as shown in Fig. 2) to ensure the symphony engineering (Fig. 5).

\section{References}

1. Huang, G.Q., Lee, S.W., Mak, K.L.: Web-based product and process data modeling in concurrent design for X. Robotics and Computer-Integrated Manufacturing 15, 53-63 (1999)

2. Demoly, F., Matsokis, A., Kiritsis, D.: A mereotopological product relationship description approach for assembly oriented design. Robotics and Computer-Integrated Manufacturing 28(1), 681-693 (2012) 
3. Demoly, F., Yan, X.-T., Eynard, B., Rivest, L., Gomes, S.: An Assembly oriented design framework for product structure engineering and assembly sequence planning. Robotics and Computer-Integrated Manufacturing 27(1), 33-46 (2011)

4. Demoly, F., Yan, X.-T., Eynard, B., Kiritsis, D., Gomes, S.: Integrated product relationships management: a model to enable concurrent product design and assembly sequence planning. Journal of Engineering Design 23(7), 544-561 (2012)

5. Demoly, F., Toussaint, L., Eynard, B., Kiritsis, D., Gomes, S.: Geometric skeleton computation enabling concurrent product engineering and assembly sequence planning. Computer-Aided Design 43(12), 1654-1673 (2011)

6. Rehman, F.U., Yan, X.T.: Supporting early design decision making using design context knowledge. Journal of Design Research 6(1-2), 169-189 (2007)

7. Object Management Group: OMG Systems Modeling Language (OMG SysML) specification, version 1.3 (2012), http://www.omg.org/spec/SysML/1.3/PDF 